\title{
Physical Activity and Adherence to Mediterranean Diet Increase Total Antioxidant Capacity: The ATTICA Study
}

\author{
Stavros A. Kavouras, ${ }^{1}$ Demosthenes B. Panagiotakos, ${ }^{1}$ Christos Pitsavos, ${ }^{2}$ \\ Christina Chrysohoou, ${ }^{2}$ Giannis Arnaoutis, ${ }^{1}$ Yannis Skoumas, ${ }^{2}$ \\ and Christodoulos Stefanadis ${ }^{2}$ \\ ${ }^{1}$ Department of Nutrition and Dietetics, Harokopio University, 17671 Athens, Greece \\ ${ }^{2}$ First Cardiology Clinic, School of Medicine, University of Athens, Athens, 11528 Athens, Greece \\ Correspondence should be addressed to Demosthenes B. Panagiotakos, d.b.panagiotakos@usa.net
}

Received 26 July 2010; Accepted 4 October 2010

Academic Editor: D. Giugliano

Copyright (๑) 2011 Stavros A. Kavouras et al. This is an open access article distributed under the Creative Commons Attribution License, which permits unrestricted use, distribution, and reproduction in any medium, provided the original work is properly cited.

\begin{abstract}
We studied the association of physical activity and adherence to the Mediterranean diet, in total antioxidant capacity (TAC). A random sample of 1514 men and 1528 women was selected from Attica region. Physical activity was assessed with a translated version of the validated "International Physical Activity Questionnaire" (iPAQ), and dietary intake through a validated Food Frequency Questionnaire (FFQ). Adherence to the Mediterranean diet was assessed by the MedDietScore that incorporated the inherent characteristics of this diet. TAC was positively correlated with the degree of physical activity $(P<.05)$. TAC was also positively correlated with MedDietScore $(r=0.24, P<.001)$. Stratified analysis by diet status revealed that the most beneficial results were observed to highly active people as compared to inactive, who also followed the Mediterranean diet $(288 \pm 70 \mu \mathrm{mol} / \mathrm{L}$, $230 \pm 50 \mu \mathrm{mol} / \mathrm{L}$, resp.), after adjusting for various confounders. Increased physical activity and greater adherence to the Mediterranean diet were associated with increased total antioxidant capacity.
\end{abstract}

\section{Introduction}

Physical activity has been evolved as a significant factor towards the prevention of cardiovascular and metabolic diseases as well as some kinds of cancer [1-5]. From early studies, however, it has been reported that strenuous physical activity induces a decrease in antioxidant levels and a concomitant increase in the markers of lipid peroxidation in target tissues and blood [6]. It is also known that vigorous exercise induces mitochondrial generation and/or leakage of superoxide and hydrogen peroxide with or without reduction in tocopherol (vitamin E) content in both muscle and liver [7]. Furthermore, from animal studies it has been found that exercise could accelerate the development of mammary tumors linked to exercise-induced oxidants production [8]. Simultaneously, the impact of a high fat diet has shown to reverse the beneficial effect of caloric restriction in oxidative stress in the muscle tissue of rats [9]. Moreover, a recent study in experimental animals fed with high fat diet showed that physical exercise did not provide any beneficial effect on oxidative stress and antioxidant defenses [10]. Lastly, in a group of 30 sportsmen, antioxidant supplements offered protection against exercise-induced oxidative stress [10].

Likewise, it is well documented from numerous epidemiological studies that diets rich in vegetables, fruits, whole grains, legumes, fish, and low-fat dairy products are associated with lower incidence of various chronic diseases [11, 12]. The dietary characteristics found in the olive growing areas of the Mediterranean region (i.e., Greece, Spain, Italy, and France) have been also associated with lower incidence of cardiovascular diseases, metabolic disorders, and several types of cancer [13-18]. Furthermore, a recent meta-analysis showed that greater adherence to a Mediterranean diet is associated with a significant improvement in health status, as seen by a significant reduction in overall mortality (9\%), mortality from cardiovascular diseases (9\%), incidence of or 
mortality from cancer (6\%), and incidence of Parkinson's disease and Alzheimer's disease (13\%). These results seem to be clinically relevant for public health, in particular for encouraging a Mediterranean-like dietary pattern for primary prevention of major chronic diseases [19]. These benefits have been associated with higher ability to cope with daily oxidative stress [20].

The determination of antioxidant capacity is a wellestablished tool in medical diagnosis and treatment of several diseases, such as cardiovascular disease, diabetes mellitus, cancer, and aging [21]. One of the most widely accepted index of whole body antioxidant ability is the total antioxidant capacity (TAC). Evaluation of TAC is one of the most common procedures employed to evaluate the hydrosoluble antioxidant status of biological fluids. Total antioxidant capacity (TAC) considers the cumulative action of all antioxidants that are present in plasma and body fluids and provides an integrated measurement rather than the simple sum of measurable antioxidants. A wide range of evidence indicates the importance of TAC in plasma and tissues, and of its practicability as a tool for investigating the association between diet and oxidative stress [22]. In a recent study, investigating the relation between TAC and obesity in children and adolescences, TAC was inversely associated with body mass index, standard deviation score of body mass index, and total body fat, only in obese subjects. These data suggest that TAC can also be used as a potential indicator of the risk to develop obesityrelated features [23]. Furthermore, data from the same group suggest that dietary TAC may be also a potential early estimate of the risk to develop metabolic syndrome features [24]. Finally, in a crossover trial where 24 subjects received a two-week diet high in antioxidant capacity, the results indicated that this type of diet improves significantly endothelial function in the volunteers at low cardiovascular risk, and may further reduce their risk of CVD [25].

Summarizing, the effect of exercise on oxidative stress seems not to be clear and the exercise effects might be modulated by other important factors like diet. Therefore, the purpose of the present study was to examine the separate and combined effect of physical activity and adherence to the Mediterranean diet on TAC.

\section{Materials and Methods}

The "ATTICA" epidemiological study has been carried out in the province of Attica (including 78\% urban and 22\% rural areas). The sampling was random, multistage by city, and stratified by age and gender group according to the gender-age distribution of the province of Attica (2001 census). From May 2001 to December 2002, 4056 inhabitants from the above area were randomly selected to enrol into the study (via mail or telephone). However, 3042 of them agreed to participate (75\% participation rate). Each participant gave informed written consent and the protocol was approved by the Medical Research Ethics Committee of Athens Medical School. All participants were interviewed by trained personnel (cardiologists, general practitioners, dieticians, and nurses) who used standard questionnaires that evaluated lifestyle habits and various sociodemographic, clinical and biological characteristics. Five percent of men and 3\% of women were excluded from the study because they had history of cardiovascular or other atherosclerotic disease, as well as chronic viral infections, as it was ascertained by their medical records. Moreover, participants did not have cold or flu, acute respiratory infection, dental problems, or any type of surgery during the past weeks.

Power analysis showed that the number of enrolled participants is adequate to evaluate two-sided standardised differences greater than 0.5 between diet subgroups and the investigated biochemical parameters, achieving statistical power greater than 0.90 at $5 \%$ probability level ( $P$-value).

The study was approved by the Medical Research Ethics Committee of Athens Medical School and was carried out in accordance with the Declaration of Helsinki (1983) of the World Medical Association.

2.1. Physical Activity Ascertainment. A translated version of the validated "International Physical Activity Questionnaire" (iPAQ), suitable for assessing population levels of selfreported physical activity was used [26]. The short version of iPAQ provided information on weekly time spent walking, in vigorous, moderate-intensity, and in sedentary activity. Participants were instructed to refer to all domains of physical activity during a usual week of the past year. Both continuous and categorical indicators of physical activity status were assessed. In particular, the continuous indicator was calculated as a sum of weekly MET-minutes per week of walking, moderate- and vigorous-intensity exercise. Furthermore, the categorical analysis grouped the subjects in three levels that were developed based on a key concept in current public health guidelines for physical activity [27], that is, (a) inactive, (b) minimally active, and (c) HEPA active (health enhancing physical activity, a high active category). The criteria of grouping in each category have been previously described [28].

2.2. Dietary Assessment. Consumption of unrefined cerealsand products, legumes, fruits, poultry, vegetables, olive oil, nonfat or low-fat dairy products, fish, nuts, potatoes, eggs, sweets, red meat, and meat product, was measured as average amounts consumed per week during the past year, with the use of a validated food frequency questionnaire (FFQ) from the department of nutritional epidemiology of Athens Medical School [29]. The frequency of consumption was then quantified approximately in terms of the number of times per month that a food was consumed. Alcohol consumption was measured by daily ethanol intake, in wine glasses ( $100 \mathrm{~mL}$ and $12 \mathrm{~g}$ ethanol concentration). Based on the Mediterranean diet pyramid [30], we calculated a special diet score ranging from 0 to 55 [31]. Higher values of this score (MedDietScore) indicate adherence to the Mediterranean diet, whereas lower values indicate adherence to the "Westernized" diet. 
2.3. Sociodemographic and Lifestyle Variables. Additionally to the physical activity and dietary status, the study's questionnaire included demographic characteristics such as, the financial status of the study's participants which was recorded as the mean annual income during the past three years and their educational level (as a proxy of social status) which was measured in years of school. Information about smoking habits was also collected using a standardized questionnaire developed for the study. Current smokers were defined as those who smoked at least one cigarette per day and former smokers were characterized as those who had stopped smoking for at least $1 \mathrm{yr}$. All other individuals were classified as nonsmokers. Occasional smokers $(<7$ cigarettes per week) were recorded and combined with current smokers due to their small sample size. For a more precise evaluation of smoking habits, the pack-years (cigarette packs per day multiplied by years of smoking) were calculated. In order to take into account various types of cigarettes consumed (i.e., light, heavy, very heavy), we used as a unit 1 cigarette with nicotine content of $0.8 \mathrm{mg}$.

\subsection{Anthropometrics, Clinical, and Biochemical Characteris-} tics. Standing height and weight were recorded, and body mass index (BMI) was calculated as weight $(\mathrm{kg})$ divided by standing height $\left(\mathrm{m}^{2}\right)$. Arterial blood pressure was measured three times, at the end of the physical examination with subject in sitting position. All participants were at least 30 minutes at rest. Patients whose average blood pressure levels were greater or equal to $140 / 90 \mathrm{mmHg}$ or were under antihypertensive medication were classified as hypertensive. Blood samples were collected from an antecubital vein between 8 to $10 \mathrm{AM}$, with the subjects in a sitting position after 12 hours of fasting and avoiding of alcohol.

TAC was measured through colorimetric test in serum (ImAnOx, Immunodiagnostik AG, Bensheim, Germany). In particular, the determination of antioxidant capacity was performed by the reaction of antioxidants in the sample with a defined amount of exogenously provided hydrogen peroxide. The intra-, interassay coefficients of variation of TAC did not exceed $2 \%$ and 5\%, respectively. Further blood lipid examinations, that is, serum total cholesterol, and low-density lipoprotein cholesterol, were measured using chromatographic enzymic method in an automatic analyzer (RA-1000, Mecon Ltd, Athens, Greece). The intra- and interassay coefficients of variation of all cholesterol levels did not exceed $4 \%$. Hypercholesterolemia was defined as total serum cholesterol levels greater than $200 \mathrm{mg} / \mathrm{dL}$ or the use of lipid lowering agents.

2.5. Statistical Analysis. Continuous variables are presented as mean values \pm standard deviation. Categorical variables are presented as absolute and relative frequencies. Associations between categorical variables were tested by the calculation of chi-squared test, while differences between categorical and several biochemical, clinical, and nutritional variables were tested by the use of Student's $t$-test and Mann-Whitney test (for the normally distributed and the skewed variables, resp.). Comparisons between TAC and tertiles of the diet score were performed using one-way Analysis of Variance, after adjusting for sex. However, due to multiple comparisons, we used the Bonferroni correction in order to account for the increase in Type I error. Multiple linear regression was applied to test the association between the MedDietScore and TAC, after controlling for several potential confounders. Colinearity between independent variables was evaluated through the condition index, while model's goodness-of-fit was graphically evaluated (standardized residuals against fitted values).

All reported $P$-values are based on two-sided tests and compared to a significance level of 5\%. SPSS 14 (SPSS Inc., Chicago, IL, USA) software was used for all the statistical calculations.

\section{Results}

The descriptive characteristics of the participants are presented separately in men and women according to the MedDietScore in Table 1 . TAC was positively correlated with diet score (rho $=0.24, P<.005$ ), indicating that greater adherence to the Mediterranean diet was associated with increased TAC levels. In particular, people in the highest tertile of diet score had higher TAC levels as compared to people in the lowest tertile. Both men and women that were grouped in the higher tertile of the diet score had greater levels of physical activity as assessed by the METs min/week. Furthermore, both male and female participants in the highest tertile of the diet score were older, had lower body mass index, lower systolic blood pressure, and lower prevalence of hypertension. No associations were found between diet score and the other blood lipids measured, current smoking, and financial status (Table 1).

Figure 1 confirms the previous relationships by presenting the correlation of physical activity categories with TAC levels, as well as the combined effect that exercise and compliance to the Mediterranean diet has on TAC. It is shown that highly active people (HEPA active) have higher TAC levels compared to less active ones. The highest TAC levels were observed among people that have both the greater adherence to the Mediterranean diet and the highest level of physical activity.

However, residual confounding may exist. Thus, the unadjusted analyses were repeated after controlling for age, sex, and body mass index (factors that are known to influence both physical activity status and TAC levels). Table 2 illustrates the association of physical activity status on TAC levels, by dietary habits, after various adjustments made. The results demonstrate that people with increased physical activity combined with close or very close adherence to the Mediterranean diet had higher levels of TAC compared to those who were inactive.

\section{Discussion}

In a sample of 3042 free-living people, it was revealed that increased physical activity in combination with greater adherence to the Mediterranean diet was associated with increased TAC levels. 
TABLE 1: Lifestyle, clinical, and biochemical characteristics of the participants, according to Mediterranean diet score.

\begin{tabular}{|c|c|c|c|c|c|c|c|}
\hline & \multicolumn{4}{|c|}{$\operatorname{Men}(n=1514)$} & \multicolumn{3}{|c|}{ Women $(n=1528)$} \\
\hline & \multirow{3}{*}{$\begin{array}{c}1 \text { st }(0-20) \\
n=504\end{array}$} & \multicolumn{5}{|c|}{ Tertile of diet score } & \multirow{3}{*}{$P^{\perp}$} \\
\hline & & 2nd (21-35) & $3 r d(36-55)$ & 1st $(0-20)$ & 2nd (21-35) & $3 \mathrm{rd}(36-55)$ & \\
\hline & & $n=505$ & $n=505$ & $n=509$ & $n=509$ & $n=510$ & \\
\hline Age (years) & $44 \pm 11$ & $42 \pm 8$ & $48 \pm 7^{* *}$ & $43 \pm 7$ & $45 \pm 7^{*}$ & $47 \pm 6^{* *}$ & .01 \\
\hline Years of education & $11 \pm 4$ & $13 \pm 4$ & $14 \pm 5$ & $9 \pm 4$ & $10 \pm 4$ & $13 \pm 3$ & .001 \\
\hline Current smoking (\%) & 49 & 46 & 44 & 40 & 39 & 38 & .21 \\
\hline Physical activity status & & & & & & & .001 \\
\hline Inactive (\%) & 63 & $26^{* *}$ & $11^{* *}$ & 70 & $22^{* *}$ & $8^{* *}$ & \\
\hline Minimally active (\%) & 64 & $26^{* *}$ & $10^{* *}$ & 71 & $21^{* *}$ & $8^{* *}$ & \\
\hline HEPA (\%) & 59 & $25^{* *}$ & $16^{* *}$ & 65 & $25^{* *}$ & $10^{* *}$ & \\
\hline METs min/week & $965 \pm 1512$ & $976 \pm 1495$ & $1429 \pm 2023^{* *}$ & $728 \pm 1262$ & $688 \pm 1027^{*}$ & $901 \pm 1281^{* *}$ & .03 \\
\hline Body mass index $\left(\mathrm{kg} / \mathrm{m}^{2}\right)$ & $27 \pm 5$ & $26 \pm 4$ & $25 \pm 5^{*}$ & $26 \pm 3$ & $24 \pm 4^{*}$ & $24 \pm 3^{*}$ & .04 \\
\hline SBP (mm Hg) & $129 \pm 17$ & $125 \pm 18^{*}$ & $125 \pm 17^{*}$ & $129 \pm 18$ & $120 \pm 18^{* *}$ & $120 \pm 19^{* *}$ & .003 \\
\hline DBP (mm Hg) & $83 \pm 11$ & $81 \pm 14$ & $80 \pm 11$ & $80 \pm 11$ & $75 \pm 12$ & $75 \pm 10$ & .25 \\
\hline Hypertension (\%) & 51 & $27^{* *}$ & $20^{* *}$ & 50 & $36^{* *}$ & $10^{* *}$ & .001 \\
\hline Total cholesterol (mg/dL) & $197 \pm 43$ & $194 \pm 41$ & $194 \pm 43$ & $196 \pm 40$ & $190 \pm 42$ & $188 \pm 47$ & .14 \\
\hline LDL-cholesterol (mg/dL) & $134 \pm 43$ & $124 \pm 38$ & $124 \pm 42$ & $126 \pm 39$ & $120 \pm 37$ & $120 \pm 41$ & .07 \\
\hline Hypercholesterolemia (\%) & 45 & 39 & 36 & 51 & 47 & 25 & .08 \\
\hline $\mathrm{TAC}(\mu \mathrm{mol} / \mathrm{L})$ & $225 \pm 33$ & $242 \pm 31^{* *}$ & $251 \pm 32^{* *}$ & $231 \pm 26$ & $239 \pm 29^{*}$ & $255 \pm 44^{* *}$ & .002 \\
\hline
\end{tabular}

TAC: total antioxidant capacity; SBP: systolic blood pressure; DBP: diastolic blood pressure; LDL: low density lipoprotein.

Data are presented as mean \pm SD and percentages.

No significant interactions were observed between tertile of diet score and sex.

${ }^{\perp}$ Probability values are derived from ANOVA and evaluate the association between tertiles of diet score and the investigated variables, after adjusting for sex.

${ }^{*} P$-value $<.05 ;{ }^{* *} P$-value $<.01$ for the comparisons between 2 nd, 3 rd tertile versus 1 st tertile of diet group.

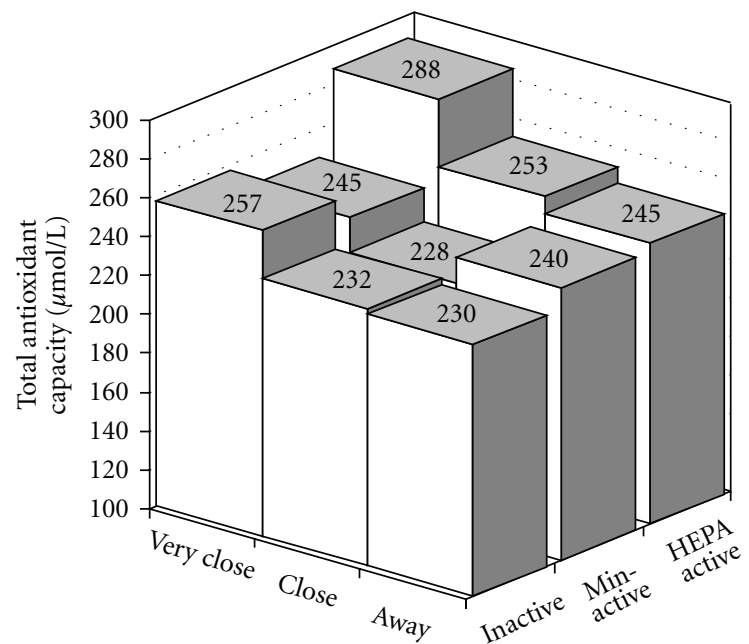

Figure 1: TAC levels and physical activity status (inactive, minactive, and HEPA active) by MedDietScore that assessed adherence to the Mediterranean diet (away, close, and very close).

These findings suggest that regular exercise in combination with this specific dietary pattern may have beneficial effect on cardiovascular system through another pathophysiological mechanism, that is, their ability to modulate oxidation process. Moreover, the results had clearly shown that highly active people who exercise several days per week enjoyed greater protection from the oxidative process due to higher TAC levels. The degree of physical activity was positively correlated with TAC, and another interesting finding of the study was that both men and women who belonged to the highly active group (HEPA active) were also those, who followed dietary patterns closer to the Mediterranean diet.

A large number of observational studies have suggested that people with greater adoption of the Mediterranean diet (i.e., high intakes of fruits, vegetables, and olive oil) experience lower risk of coronary heart disease events [3, $14,16,17,32,33]$. However, the data in changes of total antioxidant capacity (TAC) in humans is conflicting. Some investigators observed that in patients with coronary heart disease, $\alpha$-tocopherol treatment significantly reduces the rate of nonfatal myocardial infarction, while others reported that although vitamin E supplementation increased blood vitamin concentrations, it did not produce any significant reductions in the morbidity or mortality of any type of cardiovascular disease and cancer. In addition, based on a systematic review in the literature, Blomhoff [34] concluded that although observational studies have suggested that antioxidants may reduce oxidative stress, clinical trials do not support these benefits. Therefore, the scientific research 
TABLE 2: Results from multiple linear regression analysis that evaluated the association of physical activity status (independent) on TAC levels (dependent), by dietary habits.

\begin{tabular}{|c|c|c|c|}
\hline $\begin{array}{l}\text { Mediterranean } \\
\text { diet status }\end{array}$ & $\begin{array}{c}\text { Physical activity } \\
\text { status }\end{array}$ & $b \pm \mathrm{SE}$ & $P$ \\
\hline \multirow{5}{*}{ Away } & $\begin{array}{l}\text { HEPA versus } \\
\text { Inactive }\end{array}$ & $13.47 \pm 5.7$ & .21 \\
\hline & $\begin{array}{c}\text { Minimally active } \\
\text { versus inactive }\end{array}$ & $3.34 \pm 2.6$ & .48 \\
\hline & Age (years) & $-0.14 \pm 0.2$ & .58 \\
\hline & $\begin{array}{l}\text { Males versus } \\
\text { females }\end{array}$ & $-4.36 \pm 6.7$ & .52 \\
\hline & BMI $\left(\mathrm{kg} / \mathrm{m}^{2}\right)$ & $-0.32 \pm 0.64$ & .62 \\
\hline \multirow{5}{*}{ Close } & $\begin{array}{l}\text { HEPA versus } \\
\text { Inactive }\end{array}$ & $15.7 \pm 9.4$ & .05 \\
\hline & $\begin{array}{l}\text { Minimally active } \\
\text { versus inactive }\end{array}$ & $2.82 \pm 4.3$ & .09 \\
\hline & Age (years) & $-0.76 \pm 0.3$ & .03 \\
\hline & $\begin{array}{l}\text { Males versus } \\
\text { Females }\end{array}$ & $-4.85 \pm 8.2$ & .56 \\
\hline & $\operatorname{BMI}\left(\mathrm{kg} / \mathrm{m}^{2}\right)$ & $-1.79 \pm 1.33$ & .18 \\
\hline \multirow{5}{*}{ Very close } & $\begin{array}{l}\text { HEPA versus } \\
\text { Inactive }\end{array}$ & $19.7 \pm 7.4$ & .03 \\
\hline & $\begin{array}{l}\text { Minimally active } \\
\text { versus inactive }\end{array}$ & $2.4 \pm 9.6$ & .06 \\
\hline & Age (years) & $-0.16 \pm 0.3$ & .65 \\
\hline & $\begin{array}{l}\text { Males versus } \\
\text { females }\end{array}$ & $-21.8 \pm 10.7$ & .04 \\
\hline & BMI $\left(\mathrm{kg} / \mathrm{m}^{2}\right)$ & $-0.35 \pm 1.30$ & .78 \\
\hline
\end{tabular}

needs to clarify whether other plant antioxidants, or their combination, or whole dietary patterns that induce the endogenous antioxidant defense, can reduce pathogenesis of cardiovascular disease. Moreover, since there is some evidence that exhaustive exercise may have negative impact on the body's antioxidant capacity, it seems necessary to determine the level of physical activity that in combination with adaptation of a specific dietary pattern, like the Mediterranean diet, has optimal effect on TAC.

In this work it was observed that almost a $20 \%$ increment in the diet score (that assessed greater adherence to the Mediterranean dietary pattern) was associated with about $6 \%$ rise in TAC irrespective of various potential confounding factors. The high content of the Mediterranean diet in vegetables, fresh fruits, cereals, and olive oil, as well as the moderate intake of wine, guarantee a high intake of $\beta$ carotene, vitamins $\mathrm{B}_{6}, \mathrm{~B}_{12}, \mathrm{C}$, and $\mathrm{E}$, folic acid, polyphenols and various minerals, known for their antioxidant effect. However, the influence of the Mediterranean dietary pattern on antioxidant capacity of human body has been rarely investigated. To the best of our knowledge, only Leighton et al. [35] based on an intervention study, reported that total antioxidant reactivity increased by $28 \%$ above basal levels in the Mediterranean diet group, compared to the high fat diet group. In this study, we expanded the previous results since we were able to study a "free eating" population. Thus, these findings could be applicable to the general population, since the "doses" of this dietary pattern were not excessive even in the high consuming group.

From this work, it can be concluded that the combination of certain dietary patterns with an increased level of physical activity is essential for the protection of the cardiovascular system.

\section{Limitations}

Due to the cross-sectional design of the study, we cannot establish causal relations but only generate hypothesis for the association of a dietary pattern on TAC of human body. In addition, although diet is a lifelong habit and usually pre-exist a pathological condition, inverse causation may still exist. Another limitation is the misreporting of food items consumed and especially alcohol consumption, due to recall bias or social class of the participants. Moreover, the food frequency questionnaire has been validated in a sample of school teachers, while we have applied it into the general population. This may hide over- or underestimation of various nutrients estimated. However, in this work we have not used nutrient intake that may be influenced by the validation of the questionnaire.

\section{Conclusions}

Until recently, traditional analyses in the fields of nutritional epidemiology usually relate health status to a single or a few nutrients or foods. However, it is evident that people do not eat isolated nutrients, but meals consisting of a variety of foods with complex combinations of nutrients. Furthermore, the level of physical activity seems to interact with diet and both affect health status. Under this concept, the combined effect of increased physical activity with greater adherence to Mediterranean diet, on TAC was examined. It was observed that the Mediterranean diet as well frequent exercise, enhanced antioxidant defences. Alternatively, the combination of a sedentary lifestyle and a high fat diet, rich in saturated fats, induced oxidative stress.

Based on these findings, we underline the need for actions from public health care professionals, towards prevention of the development and progression of atherosclerotic diseases, through the adoption of an active lifestyle and a dietary pattern, low in fat, rich in fruits, vegetables and legumes, like the Mediterranean diet.

\section{Acknowledgments}

The authors would like to thank the field investigators of "ATTICA" study: Dr. Natasa Katinioti (physical examination), Dr. Akis Zeimbekis (physical examination), Dr. Spiros Vellas (physical examination), Dr. Efi Tsetsekou (physical/psychological evaluation), Dr. Dina Massoura (physical examination), Dr. Lambros Papadimitriou (physical examination), as well as the technical team: Dr. Marina Toutouza 
(principal investigator in biochemical analysis), Ms. Carmen Vasiliadou (genetic analysis), Mr. Manolis Kambaxis (nutritional evaluation), Ms. Konstadina Paliou (nutritional evaluation), Ms. Konstadina Tselika (biochemical evaluation), Ms. Sia Poulopoulou (biochemical evaluation) and Ms. Maria Toutouza (database management). The ATTICA study was supported by research grants from the Hellenic Cardiological Society (HCS2002). No conflict of interests or any financial disclosure exists.

\section{References}

[1] A. McTiernan, "Physical activity, exercise, and cancer: prevention to treatment-symposium overview," Medicine and Science in Sports and Exercise, vol. 35, no. 11, pp. 1821-1822, 2003.

[2] A. McTiernan, C. Kooperberg, E. White et al., "Recreational physical activity and the risk of breast cancer in postmenopausal women: the Women's Health Initiative Cohort Study," Journal of the American Medical Association, vol. 290, no. 10, pp. 1331-1336, 2003.

[3] D. B. Panagiotakos, C. Pitsavos, C. Chrysohoou, S. Kavouras, and C. Stefanadis, "The associations between leisure-time physical activity and inflammatory and coagulation markers related to cardiovascular disease: the ATTICA Study," Preventive Medicine, vol. 40, no. 4, pp. 432-437, 2005.

[4] C. Pitsavos, S. A. Kavouras, D. B. Panagiotakos et al., "Physical activity status and acute coronary syndromes survival The GREECS (Greek Study of Acute Coronary Syndromes) study," Journal of the American College of Cardiology, vol. 51, no. 21, pp. 2034-2039, 2008.

[5] C. Pitsavos, D. B. Panagiotakos, C. Chrysohoou, S. Kavouras, and C. Stefanadis, "The associations between physical activity, inflammation, and coagulation markers, in people with metabolic syndrome: the ATTICA study," European Journal of Cardiovascular Prevention and Rehabilitation, vol. 12, no. 2, pp. 151-158, 2005.

[6] K. J. A. Davies, A. T. Quintanilha, G. A. Brooks, and L. Packer, "Free radicals and tissue damage produced by exercise," Biochemical and Biophysical Research Communications, vol. 107, no. 4, pp. 1198-1205, 1982.

[7] C. K. Sen, M. Atalay, J. Ågren, D. E. Laaksonen, S. Roy, and O. Hänninen, "Fish oil and vitamin E supplementation in oxidative stress at rest and after physical exercise," Journal of Applied Physiology, vol. 83, no. 1, pp. 189-195, 1997.

[8] C. A. Gillette, Z. Zhu, K. C. Westerlind, C. L. Melby, P. Wolfe, and H. J. Thompson, "Energy availability and mammary carcinogenesis: effects of calorie restriction and exercise," Carcinogenesis, vol. 18, no. 6, pp. 1183-1188, 1997.

[9] B. Garait, K. Couturier, S. Servais et al., "Fat intake reverses the beneficial effects of low caloric intake on skeletal muscle mitochondrial $\mathrm{H}_{2} \mathrm{O}_{2}$ production," Free Radical Biology and Medicine, vol. 39, no. 9, pp. 1249-1261, 2005.

[10] R. C. M. Burneiko, Y. S. Diniz, C. M. Galhardi et al., "Interaction of hypercaloric diet and physical exercise on lipid profile, oxidative stress and antioxidant defenses," Food and Chemical Toxicology, vol. 44, no. 7, pp. 1167-1172, 2006.

[11] V. Ganji and M. R. Kafai, "Demographic, health, lifestyle, and blood vitamin determinants of serum total homocysteine concentrations in the third National Health and Nutrition Examination Survey, 1988-1994," The American Journal of Clinical Nutrition, vol. 77, no. 4, pp. 826-833, 2003.
[12] WHO/FAO, "Diet, nutrition and the prevention of chronic diseases," Tech. Rep. no. 916, Geneva, Switzerland, 2003.

[13] M. A. Martinez-Gonzalez and R. Estruch, "Mediterranean diet, antioxidants and cancer: the need for randomized trials," European Journal of Cancer Prevention, vol. 13, no. 4, pp. 327335, 2004.

[14] M. A. Martinez-González and A. Sánchez-Villegas, "The emerging role of Mediterranean diets in cardiovascular epidemiology: monounsaturated fats, olive oil, red wine or the whole pattern?" European Journal of Epidemiology, vol. 19, no. 1, pp. 9-13, 2004.

[15] H. Schröder, J. Marrugat, J. Vila, M. I. Covas, and R. Elosua, "Adherence to the traditional mediterranean diet is inversely associated with body mass index and obesity in a Spanish population," Journal of Nutrition, vol. 134, no. 12, pp. 33553361, 2004.

[16] C. Trichopoulos and P. Lagiou, "Mediterranean diet and cardiovascular epidemiology," European Journal of Epidemiology, vol. 19, no. 1, pp. 7-8, 2004.

[17] A. Trichopoulou, C. Bamia, and D. Trichopoulos, "Mediterranean diet and survival among patients with coronary heart disease in Greece," Archives of Internal Medicine, vol. 165, no. 8, pp. 929-935, 2005.

[18] A. Trichopoulou, T. Costacou, C. Bamia, and D. Trichopoulos, "Adherence to a Mediterranean diet and survival in a Greek population," New England Journal of Medicine, vol. 348, no. 26, pp. 2599-2608, 2003.

[19] F. Sofi, F. Cesari, R. Abbate, G. F. Gensini, and A. Casini, "Adherence to Mediterranean diet and health status: metaanalysis," British Medical Journal, vol. 337, article no. a1344, 2008.

[20] P. Bogani, C. Galli, M. Villa, and F. Visioli, "Postprandial antiinflammatory and antioxidant effects of extra virgin olive oil," Atherosclerosis, vol. 190, no. 1, pp. 181-186, 2007.

[21] G. Bartosz, "Total antioxidant capacity," Advances in Clinical Chemistry, vol. 37, pp. 219-292, 2003.

[22] M. Serafini and D. Del Rio, "Understanding the association between dietary antioxidants, redox status and disease: is the total antioxidant capacity the right tool?" Redox Report, vol. 9, no. 3, pp. 145-152, 2004.

[23] B. Puchau et al., "Dietary total antioxidant capacity and obesity in children and adolescents," International Journal of Food Sciences and Nutrition, vol. 61, no. 7, pp. 713-721, 2010.

[24] B. Puchau, M. A. Zulet, A. González de Echávarri, H. H. M. Hermsdorff, and J. A. Martínez, "Dietary total antioxidant capacity is negatively associated with some metabolic syndrome features in healthy young adults," Nutrition, vol. 26, no. 5, pp. 534-541, 2009.

[25] L. Franzini et al., "Food selection based on high total antioxidant capacity improves endothelial function in a low cardiovascular risk population," Nutrition, Metabolism, and Cardiovascular Diseases. In press.

[26] C. L. Craig, A. L. Marshall, M. Sjöström et al., "International physical activity questionnaire: 12-Country reliability and validity," Medicine and Science in Sports and Exercise, vol. 35, no. 8, pp. 1381-1395, 2003.

[27] W. L. Haskell, I.-M. Lee, R. R. Pate et al., "Physical activity and public health: updated recommendation for adults from the American College of Sports Medicine and the American Heart Association," Medicine and Science in Sports and Exercise, vol. 39, no. 8, pp. 1423-1434, 2007. 
[28] S. A. Kavouras, D. B. Panagiotakos, C. Pitsavos et al., "Physical activity, obesity status, and glycemic control: the ATTICA study," Medicine and Science in Sports and Exercise, vol. 39, no. 4, pp. 606-611, 2007.

[29] K. Katsouyanni, E. B. Rimm, C. Gnardellis, D. Trichopoulos, E. Polychronopoulos, and A. Trichopoulou, "Reproducibility and relative validity of an extensive semi-quantitative food frequency questionnaire using dietary records and biochemical markers among Greek schoolteachers," International Journal of Epidemiology, vol. 26, supplement 1, pp. S118-S127, 1997.

[30] W. C. Willett, F. Sacks, A. Trichopoulou et al., "Mediterranean diet pyramid: a cultural model for healthy eating," American Journal of Clinical Nutrition, vol. 61, no. 6, pp. 1402S-1406S, 1995.

[31] D. B. Panagiotakos, G. A. Milias, C. Pitsavos, and C. Stefanadis, "MedDietScore: a computer program that evaluates the adherence to the Mediterranean dietary pattern and its relation to cardiovascular disease risk," Computer Methods and Programs in Biomedicine, vol. 83, no. 1, pp. 73-77, 2006.

[32] M. De Lorgeril, P. Salen, J.-L. Martin, I. Monjaud, J. Delaye, and N. Mamelle, "Mediterranean diet, traditional risk factors, and the rate of cardiovascular complications after myocardial infarction: final report of the Lyon Diet Heart Study," Circulation, vol. 99, no. 6, pp. 779-785, 1999.

[33] C. Pitsavos, D. B. Panagiotakos, C. Chrysohoou, and C. Stefanadis, "Epidemiology of cardiovascular risk factors in Greece: aims, design and baseline characteristics of the ATTICA study," BMC Public Health, vol. 3, article no. 1, 2003.

[34] R. Blomhoff, "Dietary antioxidants and cardiovascular disease," Current Opinion in Lipidology, vol. 16, no. 1, pp. 47-54, 2005.

[35] F. Leighton, A. Cuevas, V. Guasch et al., "Plasma polyphenols and antioxidants, oxidative DNA damage and endothelial function in a diet and wine intervention study in humans," Drugs under Experimental and Clinical Research, vol. 25, no. 2-3, pp. 133-141, 1999. 


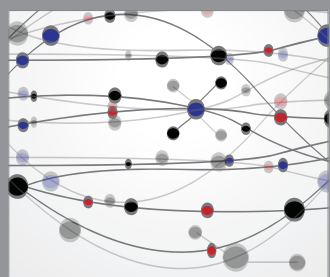

The Scientific World Journal
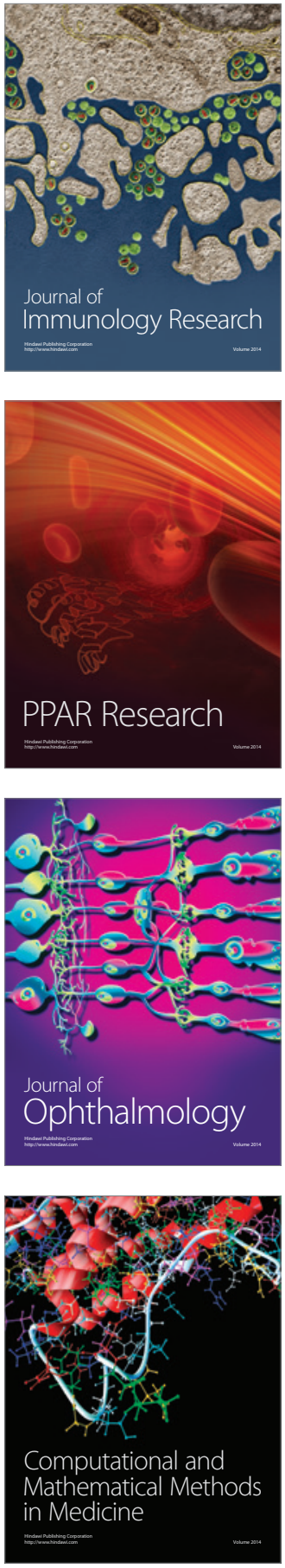



Gastroenterology

Research and Practice
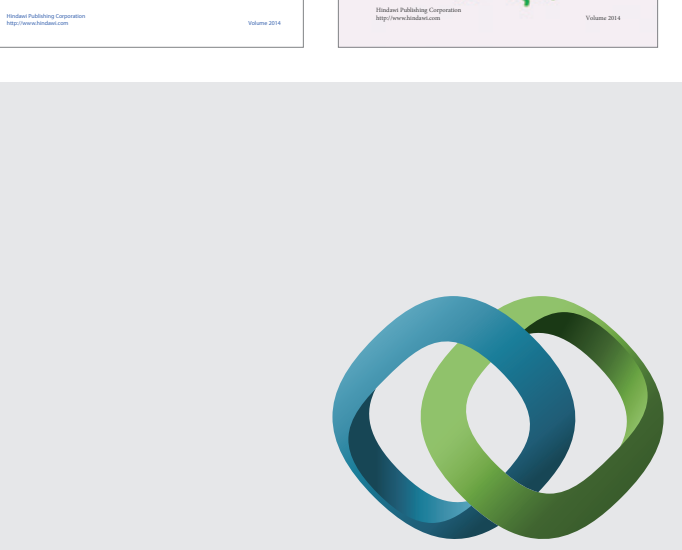

\section{Hindawi}

Submit your manuscripts at

http://www.hindawi.com
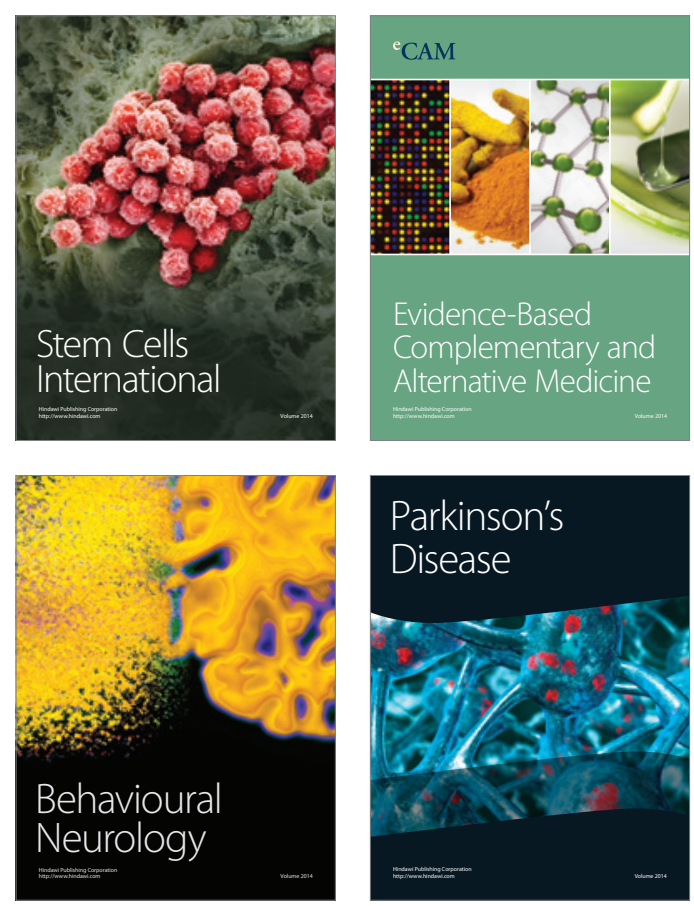



Journal of
Diabetes Research

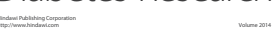

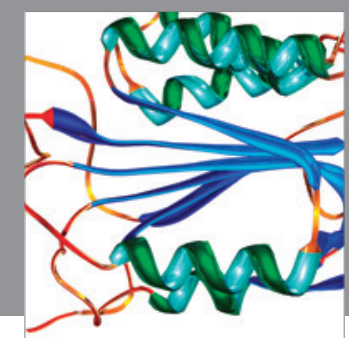

Disease Markers
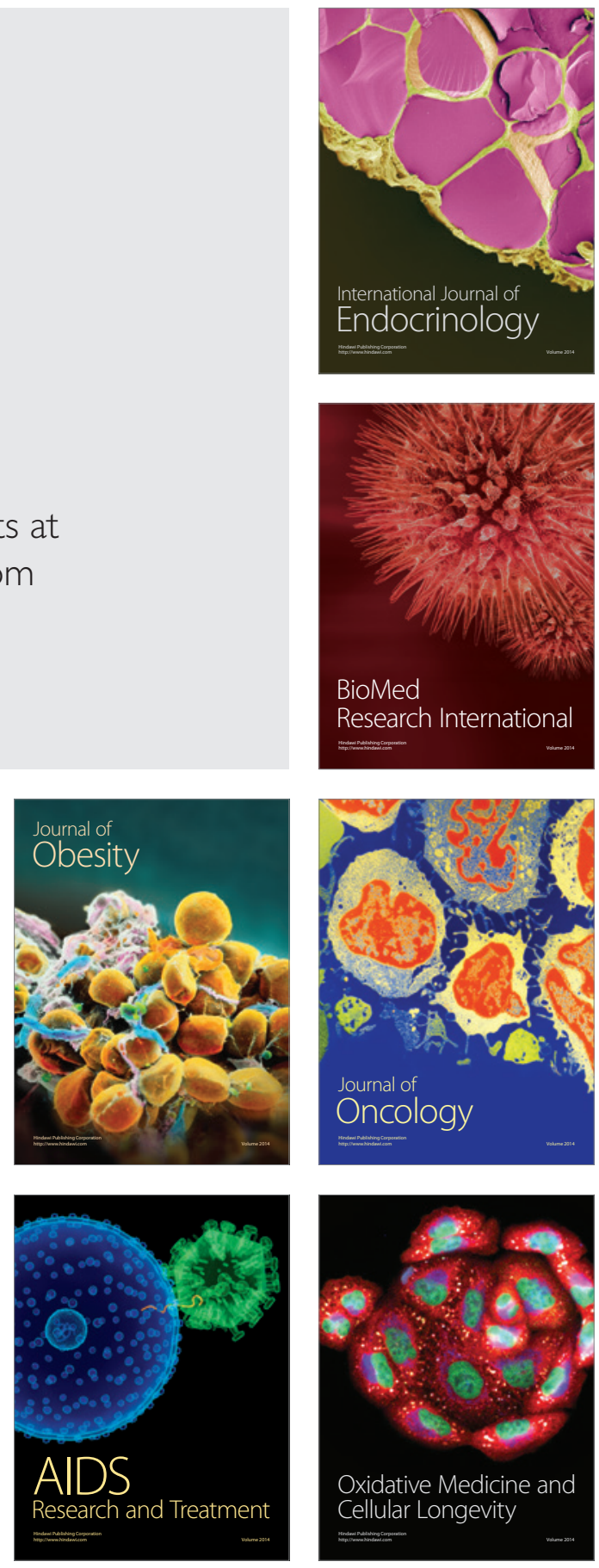